\title{
ESTUDO DE VIABILIDADE DA IMPLANTAÇÃO DE UMA LOJA DE PRODUTOS NATURAIS EM TAQUARITINGA/SP
}

\section{FEASIBILITY STUDY OF THE IMPLEMENTATION OF A NATURAL PRODUCTS STORE IN TAQUARITINGA/SP}

Ana Cristina Oliveira Nunes - ana.nunes9@fatec.sp.gov.br Faculdade de Tecnologia (Fatec) - Taquaritinga - SP - Brasil

Marcos Rafael Alves - rafael_alvesrp@hotmail.com Faculdade de Tecnologia (Fatec) - Taquaritinga - SP - Brasil

Fábio Alexandre Cavichioli - fabio.cavichioli@fatectq.edu.br Faculdade de Tecnologia (Fatec) - Taquaritinga - SP - Brasil

DOI: 10.31510/infa.v17i2.1051

Data de publicação: 18/12/2020

\section{RESUMO}

O consumo de produtos naturais está aumentando cada vez mais no contexto nacional, graças a conscientização das pessoas por alternativas que trazem benefícios e melhoria em seus hábitos alimentares. $\mathrm{O}$ trabalho apresenta a viabilidade econômica e financeira de se implantar uma loja de produtos naturais na cidade de Taquaritinga no estado de São Paulo, por meio de estimativas em três cenários (Projetado, Ruim, Péssimo). A metodologia adotada foi uma revisão bibliográfica e índices financeiros. Mapeando os gastos com o projeto foi estimado um investimento total de $\mathrm{R} \$ 50.000,00$ e um faturamento no cenário projetado de $\mathrm{R} \$ 45.000,00$ no cenário projetado, $\mathrm{R} \$ 40.500,0$ no ruim e $\mathrm{R} \$ 36.000,00$ no péssimo, tendo um custo fixo médio de $\mathrm{R} \$ 11.225,00$ e uma margem de contribuição de $69 \%$. Como resultados foi encontrado um ponto de equilíbrio de $\mathrm{R} \$ 16.268,12$ ao mês, no cenário projetado um ROI de $6.000 \%$, um retorno em três meses, um VPL de R $\$ 446.256,05$ e uma TIR de $454 \%$, já no cenário ruim um ROI de $5.011 \%$, um retorno em quatro meses, um VPL de R $\$ 365.829,70$ e uma TIR de 379\% e, no cenário péssimo um ROI de $4.032 \%$, com um retorno em cinco meses, um VPL de $\mathrm{R} \$ 285.403,34$ e uma TIR de 304\%. Apresentando viabilidade econômica e financeira em todos os indicadores e nos três cenários estimados, se caracterizando como uma excelente alternativa de investimento.

Palavras-chave: Administração Financeira. Comércio. Projeto. Viabilidade.

\begin{abstract}
The consumption of natural products is increasing increasingly in the national context, thanks to people's awareness of alternatives that bring benefits and improvement in their eating habits.
\end{abstract}


The work presents the economic and financial viability of establishing a natural products store in the city of Taquaritinga in the state of São Paulo, through estimates in three scenarios (Projected, Bad, Bad). The methodology adopted was a bibliographic review and financial indexes. Mapping the expenses with the project was estimated a total investment of $\mathrm{R} \$ 50,000.00$ and a turnover in the projected scenario of $\mathrm{R} \$ 45,000.00$ in the projected scenario, $\mathrm{R} \$ 40,500.0$ in the bad and $\mathrm{R} \$ 36,000.00$ in the bad, having an average fixed cost of $\mathrm{R} \$$ $11,225.00$ and a contribution margin of $69 \%$. As results, a break-even point of $\mathrm{R} \$ 16,268.12$ per month was found, in the projected scenario a ROI of $6,000 \%$, a return in three months, a NPV of R $\$ 446,256.05$ and a IRR of $454 \%$, already in the bad scenario an ROI of 5,011\%, a return in four months, a NPV of R $\$ 365,829.70$ and a IRR of $379 \%$ and, in the bad scenario, an ROI of $4,032 \%$, with a return in five months, a NPV of R $\$ 285,403.34$ and a $304 \%$ IRR. Presenting economic and financial viability in all indicators and in the three estimated scenarios, being characterized as an excellent investment alternative.

Keywords: Financial Administration. Store. Project. Viability.

\section{INTRODUÇÃO}

Segundo Emater (2020), a comercialização de produtos pode ser definida como o somatório de ações relacionadas a transferência de bens e serviços, desde sua origem produtiva, passando pelas cadeias produtivas indo até os clientes, e ficando à disposição dos consumidores finais. A comercialização como processo, corresponde ao conjunto de etapas ou aplicações, nas quais, produtos são transportados dos produtores aos consumidores.

Para o Portal Sebrae (2020), os comércios de produtos naturais são estabelecimentos especializados em disponibilizar itens naturais que podem ser de apenas um segmento (ofertando alimentos) ou mix de produtos contendo um leque de opções (por exemplo: produtos cosméticos, suplementos e complementos alimentares e muitos outros). Esse mix de produtos pode variar de acordo com o mercado que o empresário deseja atender e sua capacidade financeira para investir.

Em função do aumento do nicho por produtos que formam o consumo saudável e sustentável, gera ótimas possibilidades de negócios para empresários que queira atuar neste segmento se apropriando das tendências de mercado (SEBRAE, 2020). Atualmente as pessoas que moram Brasil vem se mudando seus hábitos, cuja preocupação é vida saudável. Por meio de uma pesquisa feita pela Federação das Indústrias do Estado de São Paulo, foi constatado que $80 \%$ dos entrevistados empenham para conseguir obter uma alimentação balanceada e $71 \%$ escolhem produtos naturais. 
Conforme Melo (2019), o mercado de alimentos tidos como saudáveis no país vem aumentando significativamente em função da oferta e demanda, onde as cadeias produtivas vêm se ajustando para suprir aos desejos e necessidades dos mais variados tipos de consumidores. Com um crescimento médio de aproximadamente $12 \%$ a.a.

De acordo com Menezes (2012), todo individuo ou empresa deve saber como utilizar seu dinheiro, ou seja, o mesmo pode ser poupado, gasto ou ambos. No caso de poupar uma quantia financeira é suspenso o investimento. Já ao decidir por investir o recurso (ativo fixo ou imobilizado) ou valor financeiro, o objetivo é atingir lucros, ou seja, recuperar o capital investido e obter ganhos.

O trabalho tem como objetivo principal analisar a instalação de uma loja de produtos naturais na cidade de Taquaritinga - SP que irá comercializar um mix de produtos caracterizados como naturais de forma direta ao consumidor final.

\section{FUNDAMENTAÇÃO TEÓRICA}

De acordo com o Portal Sebrae (2020), a quantidade de pessoas que buscam uma melhor alimentação vem crescendo, possibilitando o surgimento de novos negócios. Um estudo realizado pelo Sebrae aponta $80 \%$ das pessoas aceitam pagar mais caro por um alimento que lhes ofereçam maior valor nutricional, abrindo nossas possibilidades de negócios e investimentos.

Dutra et al. (2007, p. 17) estabelecem que

Uma alimentação saudável é aquela que atende todas as exigências do corpo, ou seja,
não está abaixo nem acima das necessidades do nosso organismo. Além de ser a fonte
de nutrientes, a alimentação envolve diferentes aspectos, como valores culturais,
sociais, afetivos e sensoriais. As pessoas, diferentemente dos demais seres vivos, ao
alimentar-se não buscam apenas suprir as suas necessidades orgânicas de nutrientes.
Não se alimentam de nutrientes, mas de alimentos palpáveis, com cheiro, cor, textura
e sabor, portanto, o alimento como fonte de prazer e identidade cultural e familiar
também é uma abordagem importante para promover a saúde por meio da
alimentação.

Conforme Ros, Brasioli e Guilheme (2020), ter uma boa alimentação é fundamental para manter a saúde e é indispensável para o sistema imunológico em excelentes condições e ajudando na prevenção de doenças cardiovasculares e obesidades.

Segundo SBC (2020, p. 1) “as doenças cardiovasculares - DCV's estão entre as principais causas de mortalidade no Brasil e no mundo". Elas causam cerca de 17,3 milhões de 


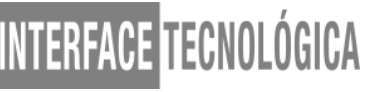

mortes por ano, onde $80 \%$ é de baixa e média renda. Logo, torna-se indispensável mostrar e orientar as pessoas adultas e crianças sobre esse problema.

Na visão de Raimundo e Machado Filho (2017, p. 10) "mantar uma alimentação saudável no dia a dia é possível e financeiramente acessível”. E precisa ser iniciada desde cedo, afinal, é durante a infância que se dá criação de hábitos alimentares, cuja participação dos pais é de suma importância para conduzir os seus filhos e crianças, reforçando campanhas de conscientização para todas as faixas etárias.

Para Dutra et al. (2007) a alimentação saudável tem como características:

$>$ Ser variada: formada por diversos tipos de alimentos, cujo objetivo é oferecer vários nutrientes (ou seja: cerais, frutas, hortaliças, carnes, laticínios e feijões).

Dstar equilibrado: levando em conta o consumo necessário de cara tipo de alimento (sendo recomendado o maior consumo de frutas em relação a gorduras).

$>$ Ter o suficiente: corresponde a quantidade de atenda e supra o mínimo para cada indivíduo.

$>$ Apresentar acessibilidade: diz respeito a alimentos in natura, plantados e vendidos na região, oferecendo preço mais competitivo e quantidade.

$>$ Oferecer cores: alimentos bem coloridos oferecem uma melhor quantidade de nutrientes, assegurando também um cardápio variado e mais atrativo, melhorando a percepção e estimulando o consumo dos itens (frutas, verduras, grãos, tubérculos e outros).

> Gerar segurança: esses itens não conter aspectos contaminantes, seja de natureza biológica, física ou química e demais perigos que possam vir a causar danos à saúde das pessoas. Por isso, é fundamental seguir os protocolos de higiene, manuseio, descarte e validade.

\subsection{Fornecedores}

De acordo com o Portal Sebrae (2020), o relacionamento com os fornecedores precisa ser sustentável. Levando em conta fornecedores da cidade ou região, cooperativas ou associações de produtores rurais. E pensar na questão ambiental, onde empresas que tem selos 
e certificações podem ser potenciais fornecedoras de mercadorias. A sustentabilidade é um ponto chave para atuar no mercado de produtos naturais e realizar negociações.

Para escolher um fornecedor deve ser analisado os seguintes aspectos (SEBRAE, 2020):

$>$ Qual a distância física?

$>\mathrm{O}$ fornecedor possui referências?

Qual o custo de frete?

> Possui qualidade mínima ou necessária?

$>$ Consegue atender a demanda de estoques em quantidade e prazo?

$>$ Qual seu preço?

$>$ Quais são as formas de pagamento?

Abaixo segue o Quadro 1 de oportunidades e ameaças de um negócio de produtos naturais que deve ser levado em consideração.

Quadro 1 - Oportunidades e ameaças em mercado de produtos naturais

\begin{tabular}{|l|ll|}
\hline \multicolumn{1}{|c|}{ OPORTUNIDADES } & \multicolumn{1}{c|}{ AMEAÇAS } \\
\hline$>$ Produtos sem glúten; & $>$ & Instabilidade econômica e políticas no país; \\
$>$ Produtos fitness; & $>$ & Baixas barreiras de entrada no varejo; \\
$>$ Produtos Veganos; & $>$ & Concorrência no varejo de alimentação. \\
$>$ Diferenciação pelos serviços. & & \\
\hline
\end{tabular}

Fonte Portal Sebrae (2020) ajustado pelos autores

\subsection{Consumidores}

Para Grassi Neto (2017), o perfil de um consumidor pode variar de acordo com suas crenças, valores, atual contexto, e aspectos psicológicos que somados irão nortear para o tipo de produto que será escolhido para sua alimentação.

De acordo com Ferreira e Carvalho (2010, p. 21 e 22) são muitos os fatores que podem influenciar durante a escolha de um produto, dentre as quais podem ser:

$>$ Motivação: Os consumidores podem ser influenciados por necessidades, que são divididas em fisiológicas (fome, sede, desconforto) e psicológicas (reconhecimento, autoestima, relacionamento), mas, muitas vezes, estas necessidades não serão fortes suficientemente para motivar a pessoa a agir num dado momento, ou seja, a pessoa às vezes necessita de um motivo maior para buscar sua satisfação.

$>$ Personalidade: É a personalidade de cada pessoa que vai determinar o seu comportamento no ato da compra, pois se refere a características psicológicas que conduzem uma resposta relativamente consistente no ambiente onde a pessoa está inserida. Os autores destacam, ainda, que o conhecimento da personalidade pode ser muito útil para analisar o comportamento do consumidor quanto a uma marca ou a um produto. 
Percepções: Consideradas como processo pelo qual as pessoas selecionam, organizam e interpretam informações para formar uma imagem significativa do mundo.

Conforme o Portal Agronovas (2019, p. 1) "o consumidor de hoje tem novo perfil na hora de escolher alimentos". Ao fazer suas compras as pessoas estão olhando de maneira sistêmica em relação ao produto saudável, indo além da ideia de somente perder peso.

Na visão do Portal MilkPoint (2018, p. 1), aponta que o perfil dos consumidores de alimentos saudáveis de acordo com uma pesquisa realizada pelos Institutos Nielsen, Kantar Wordpanel, Gfk e Ibope, apresentados na 33ª edição da Apas Show em 2017, menciona que o perfil dos consumidores são:

$>$ Mulheres e consumidores de maior idade dão mais importância a hábitos alimentares mais naturais e saudáveis;

> Dão mais importância à ausência de componentes nocivos (aditivos, conservantes, corantes, entre outros) do que à existência de componentes benéficos (vitaminas, ingredientes naturais, entre outros);

$>$ Alimentos mais procurados por esses consumidores: ricos em proteína, de origem orgânica e livre de transgênicos;

> Bebidas mais procuradas por esses consumidores: chá, chá gelado, iogurte/smoothie e bebida energética.

Segundo IBE (2020), antes eram associados somente para pessoas que praticavam esportes, academias e pacientes que precisavam de dietas, atualmente esses itens visam gerar vidas mais saudáveis e ganham mais notoriedade no cotidiano das pessoas.

\subsection{Concorrentes}

De acordo com Portal Sebrae (2020), o mercado concorrente de alimentação saudável tem um crescimento sensível no contexto nacional. E de acordo com a entidade de pesquisa Euro Monitor Internacional, até 2021 o mercado de alimentação saudável no país deve aumentar cerca de $4,41 \%$ a.a.

O segmento de comida e bebida saudável está em notoriedade, apenas em 2016, foram movimentados $\mathrm{R} \$ 93,6$ bilhões em vendas, por meio desse valor o Brasil atingiu a $5^{\mathrm{a}}$ colocação entre países de maior relevância no setor. Sendo uma ótima alternativa para montar um negócio e realizar investimento.

Conforme Eidelwein e Vieira (2016), esse é um mercado que exige dos empreendedores ótimas estratégias de diferenciação, nas quais, irão atender a diversos tipos consumidores que buscam mais e querem pagar um preço justo, onde o negócio irá atingir o retorno sobre o capital 


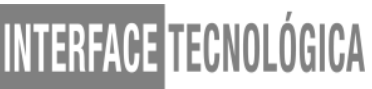

aplicado, o uso de planejamento permite atuar num mercado cheio de concorrentes e garantir a sustentabilidade empresarial.

\section{METODOLOGIA}

O trabalho foi constituído por meio de dados referenciais que são oferecidos pelo SEBRAE, e utilizando os conhecimentos das disciplinas de gestão de projetos 1,2 e 3 , analisando o negócio proposto, seus gastos (custos e investimentos) necessários e os conhecimentos de administração financeira - levando em consideração projeções de caixa e projeção de cenários (projetado, ruim - 10\% e péssimo - 20\%) durante 10 períodos, ou seja, 10 anos de projeto.

Para reforçar o trabalho foi empregada a revisão bibliográfica fornecendo sustentação para a pesquisa, mediante a consulta em livros, publicações cientificas e sites relacionados ao tema proposto.

$\mathrm{Na}$ primeira parte, verificaram-se os gastos com o projeto em investimentos fixos (máquinas, equipamentos, móveis e utensílios), investimentos pré-operacionais (reformas, regularização do negócio), investimento financeiro (capita de giro, elaboração de estoque inicial).

Em seguida, feita a projeção de fluxo de caixa dentro de um período comercial (compreendido por 12 meses) e posteriormente acumulado em 3 períodos, caracterizado por 3 anos. Essas estimativas têm o objetivo apresentar um resultado próximo ao da realidade.

E por fim, os conceitos e aplicações da administração financeira, também chamada de engenharia econômica que se apropriou dos dados para fornecer informações aderentes a realidade do projeto, vindo a fornecer alguns indicadores (Ponto de Equilíbrio - PE; Payback Descontado - PBD; Taxa Interna de Retorno - TIR; Valor Presente Líquido - VLP e Retorno sobre Investimento - ROI), sendo utilizada a planilha eletrônica do Microsoft Excel.

\subsection{Investimentos}

De acordo com o Portal Sebrae (2020), o investimento varia em função do tamanho que será feito o projeto montado, tipo e quantidade de produtos que serão comercializados. Mas os principais equipamentos precisos para montar um estabelecimento de produtos naturais são: 
Balcão de atendimento, prateleiras, telefone, móveis de escritório, computador, freezer e geladeira. Esse negócio não carece de investimento altos em tecnologia, mas é importante ter uma planilha eletrônica de controle ou software que controle de atividades.

Para esse projeto será levado em consideração uma área de $60 \mathrm{~m}^{2}$, cujo investimento estimado é de aproximadamente $\mathrm{R} \$ 50$ mil reais, que será aplicado de acordo com a Tabela 1.

Tabela 1 - Investimentos em um projeto comércio de produtos naturais

\begin{tabular}{|c|c|}
\hline INVESTIMENTO FIXO & \multirow[b]{2}{*}{$\begin{array}{l}\mathrm{R} \$ 7.000,00 \\
\mathrm{R} \$ 3.000,00 \\
\mathrm{R} \$ 10.000,00 \\
\mathrm{R} \$ 2.000,00\end{array}$} \\
\hline 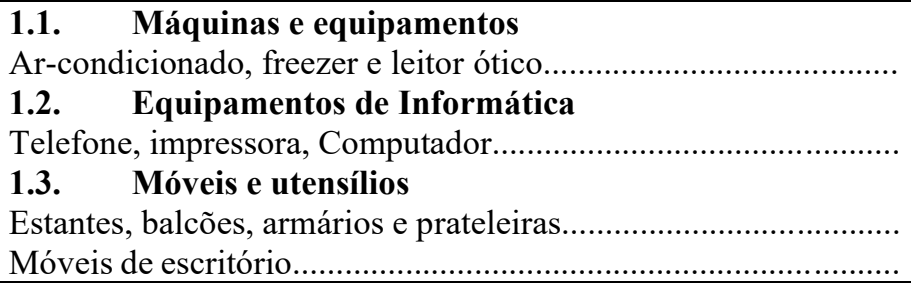 & \\
\hline 2. $\quad$ INVESTIMENTO PRÉ-OPERACIONAL & \\
\hline 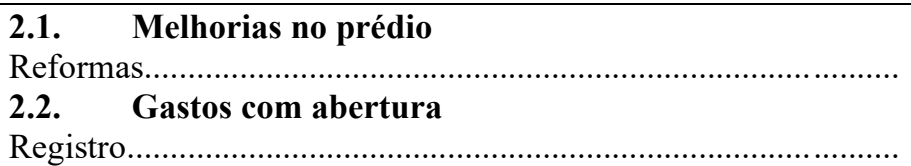 & $\begin{array}{l}\mathrm{R} \$ 10.000,00 \\
\mathrm{R} \$ 2.000,00\end{array}$ \\
\hline INVESTIMENTOS FINANCEIROS & \\
\hline 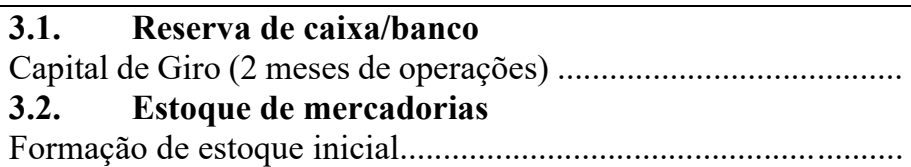 & $\begin{array}{l}\mathrm{R} \$ 7.000,00 \\
\mathrm{R} \$ 9.000,00\end{array}$ \\
\hline 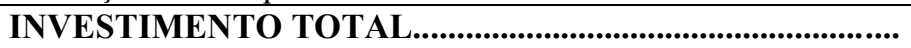 & $\mathrm{R} \$ 50.000,00$ \\
\hline
\end{tabular}

Fonte: Portal Sebrae (2020) ajustado pelos autores

Segundo o Sebrae (2020), os gastos relacionados ao negócio estão divididos em impostos, custos de mercadorias vendidas - CMV, despesas com cartão, aluguel, energia elétrica, água, telefone e internet, material de limpeza e escritório, serviços de terceiros (contabilidade, propaganda, consultoria, treinamento e software). Esses gastos são conhecidos como gastos fixos e variáveis necessários para manter a atividade empresarial e podem variar de acordo com o faturamento, contexto econômico e estratégias adotadas pela organização.

Abaixo na Tabela 2, segue o modelo de um Fluxo de Caixa - FC para o projeto levando em conta um mês de operação, utilizando os cenários projetado, ruim com redução de $10 \%$ de receita bruta e péssimo com uma redução de $20 \%$ sobre a receita.

Tabela 2 - Fluxo de caixa contendo as estimativas e possíveis cenários.

\begin{tabular}{|c|c|c|c|c|c|c|}
\hline DESCRIÇÃO DAS CONTAS & \multicolumn{2}{|c|}{ PROJETADO } & \multicolumn{2}{|c|}{ RUIM - 10\% } & \multicolumn{2}{|c|}{ PÉSSIMO - 20\% } \\
\hline RECEITA BRUTA MÊS & $\mathbf{R} \$ 45.000,00$ & $100 \%$ & $R \$ 40.500,00$ & $100 \%$ & $\mathbf{R} \$ 36.000,0$ & $100 \%$ \\
\hline (-) Imposto 9\% & $-\mathrm{R} \$ 4.050,00$ & $9 \%$ & $-\mathrm{R} \$ 3.645,00$ & $9 \%$ & $\begin{array}{l}- \\
\mathrm{R} \$ 3.240,00\end{array}$ & $9 \%$ \\
\hline (-) CMV & $-\mathrm{R} \$ 9.000,00$ & $20 \%$ & $-\mathrm{R} \$ 8.100,00$ & $20 \%$ & $\begin{array}{l}- \\
\mathrm{R} \$ 7.200,00\end{array}$ & $20 \%$ \\
\hline
\end{tabular}




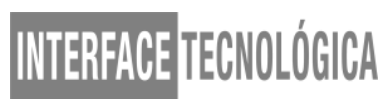

\begin{tabular}{|c|c|c|c|c|c|c|}
\hline (-) Desp Cartão Deb. e Cred. 2,5\% & -R\$ $1.125,0$ & $3 \%$ & $-\mathrm{R} \$ 1.012,50$ & $3 \%$ & $-R \$ 900,00$ & $3 \%$ \\
\hline $\begin{array}{l}\text { (=) MARGEM DE } \\
\text { CONTRIBUIÇÃO }\end{array}$ & $\mathbf{R} \$ 30.825,0$ & $69 \%$ & R\$ 27.742,50 & $69 \%$ & $\mathrm{R} \$ 24.660,0$ & $69 \%$ \\
\hline (-) GASTOS FIXOS TOTAIS & $-\mathrm{R} \$ 11.225,0$ & $25 \%$ & $-\mathrm{R} \$ 11.225,00$ & $28 \%$ & $-\mathrm{R} \$ 11.225,0$ & $31 \%$ \\
\hline (-) Aluguel & $-\mathrm{R} \$ 2.500,0$ & $5,6 \%$ & $-\mathrm{R} \$ 2.500,00$ & $6 \%$ & $-\mathrm{R} \$ 2.500,0$ & $7 \%$ \\
\hline (-) Água, Luz, telefone e internet & $-\mathrm{R} \$ 800,00$ & $1,8 \%$ & $-\mathrm{R} \$ 800,00$ & $2,0 \%$ & $-\mathrm{R} \$ 800,00$ & $2 \%$ \\
\hline $\begin{array}{l}\text { (-) Material limpeza, higiene e } \\
\text { escritório }\end{array}$ & $-\mathrm{R} \$ 500,00$ & $1,1 \%$ & $-\mathrm{R} \$ 500,00$ & $1,2 \%$ & $-\mathrm{R} \$ 500,00$ & $1 \%$ \\
\hline (-) Assessoria contábil & $-\mathrm{R} \$ 700,00$ & $1,6 \%$ & $-\mathrm{R} \$ 700,00$ & $1,7 \%$ & $-\mathrm{R} \$ 700,00$ & $2 \%$ \\
\hline (-) Propaganda & $-\mathrm{R} \$ 450,00$ & $1,0 \%$ & $-\mathrm{R} \$ 450,00$ & $1,1 \%$ & $-\mathrm{R} \$ 450,00$ & $1 \%$ \\
\hline (-) Salários, encargos e comissões & $-\mathrm{R} \$ 6.000,0$ & $13 \%$ & $-\mathrm{R} \$ 6.000,00$ & $15 \%$ & $-\mathrm{R} \$ 6.000,0$ & $17 \%$ \\
\hline (-) Depreciação mês maqui. e equip. & $-\mathrm{R} \$ 58,33$ & $0,1 \%$ & $-\mathrm{R} \$ 58,33$ & $0,14 \%$ & $-\mathrm{R} \$ 58,33$ & $0,2 \%$ \\
\hline (-) Depreciação equi. informática & $-\mathrm{R} \$ 50,00$ & $0,1 \%$ & $-\mathrm{R} \$ 50,00$ & $0,12 \%$ & $-\mathrm{R} \$ 50,00$ & $0,1 \%$ \\
\hline (-) Depreciação móveis e uten. & $-\mathrm{R} \$ 166,67$ & $0,4 \%$ & $-\mathrm{R} \$ 166,67$ & $0,41 \%$ & $-\mathrm{R} \$ 166,67$ & $0,5 \%$ \\
\hline (=) LUCRO OP. ANTES INVESTI. & R\$19.600,0 & $44 \%$ & R\$ $16.517,50$ & $41 \%$ & R\$13.435,0 & $37 \%$ \\
\hline (-) Compra de um novo Software & $-\mathrm{R} \$ 180,00$ & $0,4 \%$ & $-\mathrm{R} \$ 180,00$ & $0,4 \%$ & $-\mathrm{R} \$ 180,00$ & $0,5 \%$ \\
\hline (-) Consultoria empresarial/Marketing & $-\mathrm{R} \$ 250,00$ & $0,6 \%$ & $-\mathrm{R} \$ 250,00$ & $0,6 \%$ & $-R \$ 250,00$ & $0,7 \%$ \\
\hline (-) Treinamento pessoal & $-\mathrm{R} \$ 150,00$ & $0,3 \%$ & $-\mathrm{R} \$ 150,00$ & $0,4 \%$ & $-R \$ 150,00$ & $0,4 \%$ \\
\hline$(+/-)$ Movimentações não operacionais & $\mathrm{R} \$ \quad-$ & $0 \%$ & $\mathrm{R} \$ \quad-$ & $0,0 \%$ & $-\mathrm{R} \$ \quad-$ & $0,0 \%$ \\
\hline (=) LUCRO LÍQUIDO & $\mathbf{R} \$ 19.020,0$ & $42 \%$ & R\$ $15.937,50$ & $39 \%$ & $\mathbf{R} \$ 12.855,0$ & $36 \%$ \\
\hline
\end{tabular}

Fonte: Portal Sebrae (2020) ajustado pelos autores.

Com isso, no cenário projetado com uma receita líquida de $\mathrm{R} \$ 19.020,00$ por 12 meses será atingido um total de $\mathrm{R} \$ 228.240,00$ ao ano, já no cenário ruim o faturamento mensal é de $\mathrm{R} \$ 15.937,50$ e acumulado em 12 meses será de $\mathrm{R} \$ 191.250,00$ ao ano e por último no cenário péssimo o faturamento médio é de $\mathrm{R} \$ 12.855,00$ e multiplicado por um ano será de $\mathrm{R} \$ 154.260,00$.

\section{ANÁLISE DE VIABILIDADE}

Segundo Hashimoto (2020), analisar os indicadores de viabilidade do negócio possibilita ao empreendedor saber se o seu negócio terá possibilidade de sucesso e se seus recursos são suficientes. Para montar um negócio são necessários recursos (econômicos e financeiros) e tempo. Logo para adentrar no mundo do empresarial, os indivíduos precisam de segurança em relação ao investimento e entender a possibilidade de gerar lucros.

De acordo com Muniz (2012) o cálculo do ponto de equilíbrio é encontrado pela divisão do total de gastos fixos pelo percentual da margem de contribuição. Que irá acusar o mínimo necessário para se vender e não fica em prejuízo, atingindo pelo menos um resultado nulo ou superior dando lucro para o negócio. 
Nos três cenários o custo fixo é o mesmo, sendo de $\mathrm{R} \$ 11.225 .00$, porém o percentual de Margem de contribuição é de 0,69 , logo o ponto de equilíbrio pode ser visto da equação 1 abaixo.

$$
(\mathrm{R} \$ 11.225,00 / 0,69)=\mathrm{R} \$ 16.268,12 \quad \text { Equação } 1
$$

Para esse negócio o mínimo a ser vendido para cobrir os gastos fixos é o equivalente a $\mathrm{R} \$ 16.268,12$ por mês, trabalhando com uma margem de contribuição média de 0,69.

Conforme Mei (2014), em projetos comuns, é normal utilizar os seguintes indicadores financeiros: $\mathrm{ROI}^{1}, \mathrm{VPL}^{2}, \mathrm{TIR}^{3}$, Payback Descontado ${ }^{4}$ e uma $\mathrm{TMA}^{5}$ para satisfazer a necessidade de informações do empreendedor.

Neste projeto será adotada uma TMA de levando em conta risco de $10 \%$, acrescentado a liquidez de $8,6 \%$ e apropriando o rendimento da poupança $1,4 \%$, totalizando $20 \%$. a.a.

Faria (2020) aponta que para calcular o ROI de um projeto é preciso levar em consideração o ganho total acumulado no período menos o investimento, dividido pelo investimento e multiplicado por 100. A equação 2 apresenta os resultados de ROI no cenário projetado, na equação 3 o cenário ruim e posteriormente a equação 4 o ROI pessimista.

Equação 2

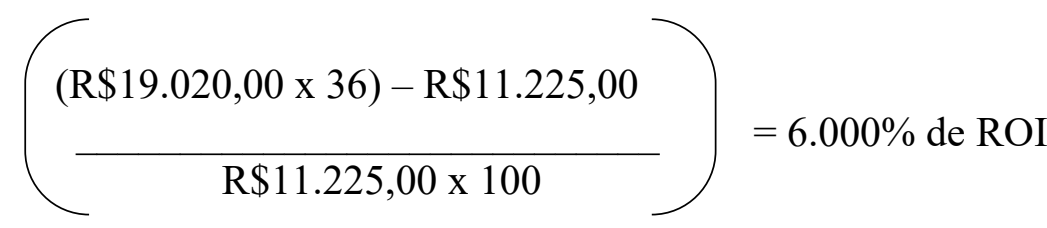

Equação 3

$$
\left(\frac{(\mathrm{R} \$ 15.937,50 \times 36)-\mathrm{R} \$ 11.225,00}{\mathrm{R} \$ 11.225,00 \times 100}\right)=5.011 \% \mathrm{de} \mathrm{ROI}
$$

\footnotetext{
${ }^{1}$ Estabelece o retorno de um investimento de forma percentual ou acumulada, sobre o valor investido.

${ }^{2}$ É um indicador que aponta o valor presente líquido que indica o retorno ajustado pela TMA, subtraído pelo investimento inicial.

3 Corresponde a Taxa Interna de Retorno do negócio, ou simplesmente uma taxa que ao modificar pela TMA, acarreta no retorno descontado igual ao investimento inicial.

${ }^{4}$ Diz respeito ao período em tempo que normalmente pode ser expresso em meses, anos que o investimento leva para ser recuperado.

${ }^{5}$ A TMA - Taxa Mínima de Atratividade é um percentual fundamental para a avaliação de investimento. Na qual, aponta o quanto de retorno uma aplicação deve remunerar.
} 


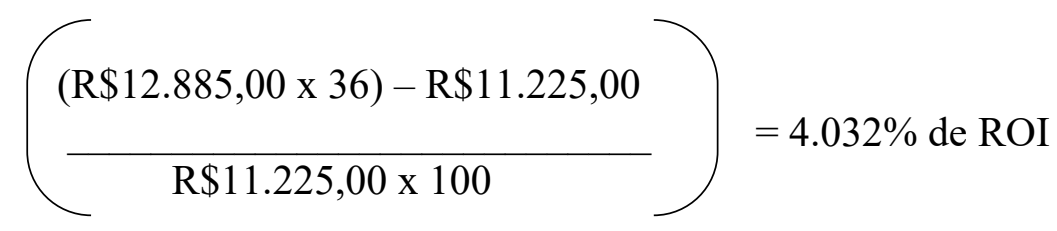

O Payback descontado foi elaborado pela planilha eletrônica Excel, conforme a Tabela 3 abaixo. Apresentando como referência os meses, Fluxo de Caixa - FC Projetado, FC a Valor Presente - VP e os saldos. A TMA de $18 \%$ a.a. foi ajustada ao mês dando um valor de $1,388 \%$ a.m.

Tabela 3 - Três cenários de projeção para Payback

\begin{tabular}{|c|c|c|c|c|c|c|c|c|c|}
\hline Mês & $\begin{array}{c}\text { F.C. } \\
\text { Projetado } \\
\end{array}$ & F.C P. a VP & $\begin{array}{c}\text { Saldo } \\
\text { Projetado } \\
\end{array}$ & F.C. Ruim & $\begin{array}{c}\text { F.C. Ruim a } \\
\text { V.P. }\end{array}$ & Saldo Ruim & F.C. Péssimo & $\begin{array}{c}\text { F.C. Pess. a } \\
\text { VP }\end{array}$ & Saldo Péssimo \\
\hline $\mathbf{0}$ & $-\mathrm{R} \$ 50000,00$ & $-\mathrm{R} \$ 50000,00$ & $-\mathrm{R} \$ 50000,00$ & $-\mathrm{R} \$ 50000,00$ & $-\mathrm{R} \$ 50000,00$ & $-\mathrm{R} \$ 50000,00$ & $-\mathrm{R} \$ 50000,00$ & $-\mathrm{R} \$ 50000,00$ & $-\mathrm{R} \$ 50000,00$ \\
\hline 1 & $\mathrm{R} \$ 19020,00$ & $\mathrm{R} \$ 18759,62$ & $-\mathrm{R} \$ 31240,38$ & $\mathrm{R} \$ 15937,50$ & $\mathrm{R} \$ 15719,32$ & $-\mathrm{R} \$ 34280,68$ & $\mathrm{R} \$ 12855,00$ & $\mathrm{R} \$ 12679,02$ & $-\mathrm{R} \$ 37320,98$ \\
\hline 2 & $\mathrm{R} \$ 19020,00$ & $\mathrm{R} \$ 18502,80$ & $-\mathrm{R} \$ 12737,59$ & $\mathrm{R} \$ 15937,50$ & $\mathrm{R} \$ 15504,12$ & -R\$18 776,57 & $\mathrm{R} \$ 12855,00$ & $\mathrm{R} \$ 12505,44$ & $-\mathrm{R} \$ 24815,54$ \\
\hline 3 & $\mathrm{R} \$ 19020,00$ & $\mathrm{R} \$ 18249,49$ & $\mathrm{R} \$ 5511,91$ & $\mathrm{R} \$ 15937,50$ & $\mathrm{R} \$ 15291,87$ & -R\$ 3484,70 & $\mathrm{R} \$ 12855,00$ & $\mathrm{R} \$ 12334,24$ & $-\mathrm{R} \$ 12481,30$ \\
\hline 4 & $\mathrm{R} \$ 19020,00$ & $\mathrm{R} \$ 17999,66$ & $\mathrm{R} \$ 23511,57$ & $\mathrm{R} \$ 15937,50$ & $\mathrm{R} \$ 15082,52$ & $\mathrm{R} \$ 11597,82$ & $\mathrm{R} \$ 12855,00$ & $\mathrm{R} \$ 12165,38$ & $-\mathrm{R} \$ 315,92$ \\
\hline 5 & $\mathrm{R} \$ 19020,00$ & $\mathrm{R} \$ 17753,24$ & $\mathrm{R} \$ 41264,81$ & $\mathrm{R} \$ 15937,50$ & $\mathrm{R} \$ 14876,04$ & $\mathrm{R} \$ 26473,87$ & $\mathrm{R} \$ 12855,00$ & $\mathrm{R} \$ 11998,84$ & $\mathrm{R} \$ 11682,92$ \\
\hline 6 & $\mathrm{R} \$ 19020,00$ & $\mathrm{R} \$ 17510,20$ & $\mathrm{R} \$ 58775,02$ & $\mathrm{R} \$ 15937,50$ & $\mathrm{R} \$ 14672,39$ & $\mathrm{R} \$ 41 \quad 146,26$ & $\mathrm{R} \$ 12855,00$ & $\mathrm{R} \$ 11834,58$ & $\mathrm{R} \$ 23517,50$ \\
\hline 7 & $\mathrm{R} \$ 19020,00$ & $\mathrm{R} \$ 17270,49$ & $\mathrm{R} \$ 76045,50$ & $\mathrm{R} \$ 15937,50$ & $\mathrm{R} \$ 14471,53$ & $\mathrm{R} \$ 55617,78$ & $\mathrm{R} \$ 12855,00$ & $\mathrm{R} \$ 11672,56$ & $\mathrm{R} \$ 35190,06$ \\
\hline
\end{tabular}

No cenário projetado o retorno ocorre por volta de 3 meses. O cenário tido como ruim, onde a projeção de vendas é negativa em 10\% o retorno ocorre em quatro meses. E por fim, no cenário péssimo de vendas negativas em $20 \%$ é atingido o retorno em cinco meses.

Ainda no projeto foi acumulado os 36 meses, dando três períodos em anos que podem ser expressos na Tabela 4, apresentando a TMA, o VPL e a TIR do projeto, devidamente processados pelas funções automáticas da planilha.

Tabela 4 - Fluxo de caixa em três cenários apresentando o VLP e TIR

\begin{tabular}{|r|c|c|c|}
\hline \multicolumn{1}{|c|}{ Ano } & Projetado & Ruim & Péssimo \\
\hline 0 & $-\mathrm{R} \$ 50000,00$ & $-\mathrm{R} \$ 50000,00$ & $-\mathrm{R} \$ 50000,00$ \\
\hline 1 & $\mathrm{R} \$ 228240,00$ & $\mathrm{R} \$ 191250,00$ & $\mathrm{R} \$ 154260,00$ \\
\hline 2 & $\mathrm{R} \$ 228240,00$ & $\mathrm{R} \$ 191250,00$ & $\mathrm{R} \$ 154260,00$ \\
\hline 3 & $\mathrm{R} \$ 228240,00$ & $\mathrm{R} \$ 191250,00$ & $\mathrm{R} \$ 154260,00$ \\
\hline \multicolumn{4}{|c|}{} \\
\hline TMA & $18 \%$ & & \\
\hline VLP & $\mathrm{R} \$ 446256,05$ & $\mathrm{R} \$ 365829,70$ & $\mathrm{R} \$ 285403,34$ \\
\hline TIR & $454 \%$ & $379 \%$ & $304 \%$ \\
\hline
\end{tabular}

Fonte: Elaborado pelos autores 


\section{CONSIDERAÇÕES FINAIS}

Ao mapear os gastos relacionados ao projeto, os indicadores de viabilidade, as informações apresentam a possibilidade de realizar um investimento, afinal, todos os índices apresentam viabilidade em tempo de 3 até 5 meses para recuperar o capital investido em tempo.

Por se tratar de um projeto de 36 meses ou 3 anos, o Valor Presente Líquido - VPL e taxa Interna de Retorno - TIR são respectivamente de $\mathrm{R} \$ 446.256,05$ e 454\% no projetado; de $\mathrm{R} \$ 365.829,70$ e $379 \%$ no ruim e $\mathrm{R} \$ 285.403,34$ e $304 \%$.

Com isso, o projeto mostra que os produtos naturais podem meio de seu alto valor agregado são uma ótima alternativa de investimento, desde que seja planejado e feito com atenção e controle, tendo a possibilidade de atender uma ótima fatia de mercado na cidade e remunerar o capital empregado com um baixo custo e alta lucratividade, conforme é apresentado no trabalho.

\section{REFERÊNCIAS BIBLIOGRÁFICAS}

DUTRA, E. S. et al. Modulo 11: Alimentação saudável e sustentável. Brasília: UNB, 2007. $92 \mathrm{p}$.

EIDELWEIN, M. D.; VIERA, E. P. Estratégias de posicionamento de mercado na comercialização de produtos naturais. Ijuí: Universidade Regional do Noroeste do Rio Grande do Sul, 2016. Disponível em: https://bibliodigital.unijui.edu.br:8443/xmlui/bitstream/handle/123456789/3906/Maiara\%20D aiane\%20Eidelwein.pdf?sequence=1\&isAllowed=y. Acesso em 23 de ago. de 2020.

EMATER. Empresara de Assistência Técnica e Extensão Rural. Conceitos de Mercado. UFRB. 19 de out. 2020. Disponível em: https://www.ufrb.edu.br/proext/images/conceitosmercado.pdf. Acesso em 19 de ago. de 2020.

FARIA, A. M. Promoção, planejamento de vendas e relacionamento com o cliente. São Paulo: Editora Senac, 2020.

FERREIRA, V. H. M.; CARVALHO, D. S. F. Comportamento do consumidor. Palhoça: UNISULVIRTUAL, 2010.

FIESP. A mesa dos brasileiros. publicado: 14 de fev. de 2019. Disponível em:https://www.fiesp.com.br/fiespcast/debate-a-mesa-dos-brasileiros/. Acesso em: 22 de ago. de 2020. 
GRASSI NETO, R. Segurança alimentar da produção agrária à proteção do consumidor. São Paulo: Saraiva, 2017.

HASHIMOTO, M. Empreendedorismo: Plano de negócios em 40 lições. $2^{\mathrm{a}}$. Ed. São Paulo: Saraiva Educação, 2020.

IBE. NOVO PERFIL DO CONSUMIDOR MOVIMENTA O MERCADO DA SAUDABILIDADE. 26 de abr. 2016. Disponível em: https://www.ibe.edu.br/novo-perfil-doconsumidor-movimenta-o-mercado-da-saudabilidade/. Acesso em: 22 de ago. de 2020.

MEI, P. C. Gerenciamento da integração em projetos. Rio de Janeiro: Elsevier, 2014.

MELO, P. Empreender: mercado de alimentação saudável cresce no Brasil. Conselho Federal de Administração. 25 de jan. de 2019.

MENEZES, M. de. Matemática financeira. Curitiba. IESDE, 2012. 328p.

MUNIZ JR, J. et al. Administração da produção. Curitiba: IESDE Brasil, 2012.

Portal Agronovas. O NOVO PERFIL DO CONSUMIDOR DE ALIMENTOS. 16 de abr. de 2019. Disponível em: http://www.agronovas.com.br/o-novo-perfil-do-consumidor-dealimentos/. Acesso em 22 de ago. de 2020.

Portal MilkPoint. Como explorar o mercado de alimentação saudável no Brasil? 15 de fev. 2018. Disponível em: https://www.milkpoint.com.br/noticias-e-mercado/gironoticias/saudabilidade-e-bemestar-uma-tendencia-no-mercado-de-alimentacao-206830/.

Acesso em 23 de ago. de 2020.

PORTAL SEBRAE. Como montar uma loja de produtos naturais. BRASILIA, DF. Disponível em: https:/www.sebrae.com.br/sites/PortalSebrae/ideias/como-montar-uma-lojade-produtos-naturais,e5297a51b9105410VgnVCM1000003b74010aRCRD\#/this. Acesso em 19 ago. 2020.

RAIMUNDO, M. G. M.; MACHADO FILHO, J. V. Alimentação saudável mais cor e sabor no seu prato. Coordenadoria de Desenvolvimento dos Agronegócios, 2017.

ROS, D. C.; BRASIOLI, M.; GUILHERME, R. C. Guia para uma alimentação saudável em tempos de Covid19. mar. de 2020. ASBRAN. Associação Brasileira de Nutrição. Disponível em: https://www.asbran.org.br/storage/downloads/files/2020/03/guia-alimentar-covid-19.pdf. Acesso em 20 de ago de 2020.

SBC. Sociedade Brasileira de Cardiologia. Manual para educadores. Programa de alimentação Saudável. Disponível em: http://cientifico.cardiol.br/sbcvaiaescola/pdf/revistaprofessores.pdf. Acesso em 20 de ago. 2020. 